\title{
IDMC-6 - The Sixth International Myotonic Dystrophy Consortium Meeting
}

\author{
G. Meola
}

\author{
Chairman and organizer IDMC-6 Università degli Studi di Milano, Dipartimento di Neurologia IRCCS \\ Policlinico San Donato, Milano, Italy
}

In 1992 three groups of investigators found that myotonic dystrophy of Steinert, classical myotonic dystrophy, now known as myotonic dystrophy type 1 $(D M 1)$, results from an unstable CTG repeat expansion in the non-coding $3^{\prime}$ region of serinethreonine kinase gene DMPK at 19q13.3. Almost a decade later in 2001 collaborators in Minnesota and Germany discovered a second gene defect responsible for myotonic dystrophy type 2 (DM2), another autosomal dominant, multisystem disease, that is similar to but distinct from DM1. DM2 results from an unstable CCTG repeat expansion in intron 1 of the zinc finger protein 9 gene ZNF9 at 3q21. The exact cause for the instability of the DNA at these two different loci, and the explanation for the tissue mosaicism that exists in different tissues in DM1 and DM2 remains a mystery although part of the answer probably lies in faulty repair of DNA. Why the CTG repeat expansion in DMI is larger in skeletal and cardiac muscle and in certain parts of the brain than in circulating leucocytes and some other tissues is not clear. Whether this tissue mosaicism accounts for or plays a major role in determining the spectrum of disease manifestations requires further investigation. Whether it is possible to use the tissue instability of DNA in refining the diagnosis and prognosis of patients with DM1 and DM2 is a question that has yet to be explored.

The overarching goal of IDMC (International Myotonic Dystrophy Consortium Meeting) is to bring together researchers, clinicians, other care providers, and patients and family members along with representatives of non-profit funding agencies and industry, and members of advocacy groups, to discuss current research discoveries, new therapeutic approaches, and optimal clinical care for patients with myotonic dystrophy. Researchers and clinicians having special interest and expertise in myotonic dystrophy will serve as co-chairs of Scientific Sessions during the meeting.

The 6th International Myotonic Dystrophy
Consortium Meeting (IDMC-6) has been organized at main campus of University of Milan, Italy, on September 12-15, 2007. All information related to this meeting are available on web page: http://www.idmc-6.com.

The IDMC-6 has followed on previous highly successful meetings IDMC-5 in Quebec (2005), IDMC-4 in Glasgow (2003), IDMC-3 in Kyoto (2001), IDMC-2 in Chapel Hill (1999) and IDMC1 in Paris (1997). IDMC-6 has been hold for the first time in Italy with the aim that remarkable progress has been achieved in the research of myotonic dystrophies (DM) and its related issues, which is expected to contribute to the better understanding of the pathomolecular mechanisms underlying physiopathogenesis of DM and the development of new innovative therapeutic approaches.

In IDMC-6, 151 abstracts at high scientific level have been submitted, which were divided, according to the International Committee Referees, into 71 oral Communications and 80 Poster presentations.

In the meeting there was the participation of a large number of young investigators and researchers (around 120), coming from Australia, Canada, Costa Rica, Cyprus, East Europe, Finland France, Germany, Israel, Italy, Japan, Russia, Scandinavian, Spain, The Netherlands, UK and USA, which guaranteed an excellent and qualified meeting. The participation of 20 outstanding chairmen of the sessions, expert in their specific fields of DM, coming from USA, UK, France, Germany, Italy and Japan, allowed continuous exchange, fruitful discussion between the authors and the attendants both in oral and poster sessions.

The total number of attendants, including investigators, physicians, patients and representative of advocacy groups was about 350 people.

The timetable scientific part of the meeting allowed on September 12 a Satellite Symposium on Genome-wide strategy in DM with the aim to collect clinical investigators for DNA studies on large scale. 
A lecture on Phenotype, genotype and molecular basis of Myotonic Dystrophy: past, present and future describing the first clinical aspect of DM 1 in the beginning of last century, the discovery of CTG repeat expansion as molecular basis of DMI and CCTG repeat expansion as molecular basis of DM2 and the innovative approach of research in DM by animal and cell models, has concluded the first day of the meeting.

On the following days (on September 13 and 14 morning), the meeting has been scheduled in basic science sessions ( 3 oral and 3 poster) with topics DNA instability and models of myotonic dystrophies, Cellular and molecular aspects of myotonic dystrophies and Rna-binding proteins (Cardani et al., 2004).

From all these basic sessions, it was very evident that animal models (transgenic mice) and cell models (myoblast, myotube) represent the best way to understand the pathomolecular mechanisms of DM.

From the meeting it was clear that none of the existing animal model of DMI closely resemble human DMI. In particular, they do not develop progressive focal muscle wasting and weakness. To clarify the mechanisms underlying the instability of DNA in both DM1 and DM2 further research is necessary, including the development of new animal models that more closely represent the clinical features of the human disorders.

Another important point is represented by the big role of RNA-binding proteins in the pathogenesis of $D M$, so these diseases are now grouped in new emerging category of diseases, due to toxic-RNA.

Results of studies in both animal models and in human tissue samples have indicated that DMI and DM2 have similar alterations in the regulation of splicing of specific proteins, such as, the skeletal muscle chloride channel, and the insulin receptor.

DM1 and DM2 are now termed splicopathies (Osborne and Thornton, 2006). Exactly how the alterations in splicing occur and how they relate to cellular and molecular genetic functions are under active investigation.

Whether the effects of the splicopathy, such as, reduced chloride channel conductance in skeletal muscle and the insulin resistance (as well as other alterations caused by the defect in splicing) (Cho and Tapscott, 2007), lead to metabolic derangements that exacerbate disease manifestations, such as, muscle wasting and fatigue, require further investigation.

From this session (RNA-binding protein), it was evident a common pathomechanism for DMI and DM2. This finding came from studies showing that several forms of the RNA binding protein muscleblind (MBNL, MBLL, MBXL) co-localize to the repeat-containing foci in tissue specimens from both DM1 and DM2 (Cardani et al., 2006). However, additional investigations in animal models and in human tissues are needed to clarify the role of the different RNA-binding proteins in the pathomechanism of DMI and DM2.

On September 14 afternoon and September 15, the meeting was focused on clinical aspects and on management of the disease ( 4 oral and 4 poster sessions), with topics Characterization and Management of systemic aspects of myotonic dystrophies, Special aspects of management and Therapeutic trials and future advances.

From all these sessions, it was evident that developing a more detailed understanding of the natural history of disease manifestations in the myotonic dystrophies (DMI and DM2) and establishing whether there was a direct relationship between the size of the CTG and CCTG expansions in affected target tissues and the severity of disease manifestations were major ongoing opportunities for clinical research, now that DNA testing is available to establish the diagnosis in patients with both conditions. Studies characterizing disease manifestations in brain, heart, respiratory muscle, gastrointestinal tract and the endocrine system, emphasized the importance that the systemic manifestations had in the lives of patients. General guidelines for management were available, but there was still limited understanding of the pathophysiologic basis for the multi-system alterations. There was a strong need for additional translational and clinical research.

Complications of pregnancy and perinatal problems, frequent in myotonic dystrophy, especially in DMI were also presented. Genetic counselling of at risk families was an essential part of management. Recent advances in prenatal diagnostic testing, made possibly largely due to the availability of DNA analysis for DMI and DM2, were presented at IDMC-6 in order to provide the most current information for researchers, clinicians, other care providers, patients and their families.

The successful trials of mexiletine for myotonia and the safety and tolerability of recombinant Human Insulin like Growth Factor 1 complexed 
with IGF Binding protein 3 (rhIGFI/IGFBP3) have been presented. As future advances, very promising findings about silencing oligo-nucleotide in myoblast - myotube model and correction of CIC-1 splicing in mouse model have been also shown.

Presentation of these findings and discussion of these translations into clinical trials in patients, was of great interest to all attendees.

At the end of the meeting (Saturday 15 afternoon), for the first time at IDMC, an interactive session between investigators, physicians, patients and representative of advocacy groups has been organized to discuss the patient's experiences, the guidelines and standard of care for DM.

The highlight session, summarizing the main aspects of research and therapeutic approach in DM during the meeting, has closed the IDMC-6 in a friendly atmosphere.

In conclusion the IDMC-6 meeting has been an excellent and outstanding meeting very interactive and will be followed by IDMC-7, scheduled on 2009 in Wurzburg - Germany, in honour of Steinert who made the first description of myotonic dystrophy last century on 1909.

\section{Acknowledgments}

Special thank to Prof. R.T. Moxley III University of Rochester (NY) - USA for strong support and advice in the organization of IDMC-6

\section{References}

Cardani R, Mancinelli E, Sansone V, Rotondo G, Meola G. Biomolecular identification of (CCTG)n mutation in myotonic dystrophy type 2 (DM2) by FISH on muscle biopsy. Eur J Histochem 2004;48:43742.

Cardani R, Mancinelli E, Rotondo G, Sansone V, Meola G. Muscleblind-like protein 1 nuclear sequestration is a molecular pathology marker of DM1 and DM2. Eur $\mathrm{J}$ Histochem 2006;50:177-82.

Cho D, Tapscott SJ. Myotonic dystrophy: emerging mechanisms for DMI and DM2. Biochemica et Biophysica Acta 2007;1772:195204.

Osborne RJ, Thornton CA. RNA-dominant diseases. Hum Mol Genet 2006;15:R162-R169.

Giovanni Meola Chairman and organizer IDMC-6 Università degli Studi di Milano Dipartimento di Neurologia IRCCS Policlinico San Donato

Via Morandi, 30 20097 San Donato Mil. (MI), Italy Phone-Fax: +39.02.52774480 E-mail:giovanni.meola@unimi.it 